\title{
Particle Candidates of Ultrahigh Energy Cosmic Rays*
}

\section{G. Domokos ${ }^{\dagger}$ and S. Kovesi-Domokos}

Department of Physics and Astronomy, The Johns Hopkins University

Baltimore, MD 21218

E-mail: :skd@jhu.edü

ABSTRACT: We discuss candidates for trans-GZK cosmic rays observed in a variety of detectors. Three types of primaries are represented among the abstracts submitted to this meeting: neutrinos causing a Z-burst, protons arising from the decay of ultra-heavy metastable particles and neutrinos within the framework of low scale string-like models of unification. We attempt to evaluate the relative merits of these schemes. No definite conclusion can be reached at this time. However, we point out that some schemes are more credible/predictive than others. Data to be gathered by the Pierre Auger observatories as well as orbiting detectors (OWL, Airwatch...) should be able to decide between the various schemes.

\section{Introduction}

As soon as the cosmic microwave backround radiation (CMBR) was discovered, Greisen and Zatsepin and Kuzmin independently pointed out that there should exist a cutoff in the primary cosmic ray spectrum ii . The physical basis of that cutoff is very well established. It is generally believed that the primary cosmic radiation at energies above, say, $10^{17.5} \mathrm{eV}$ or so, consists mostly of protons. The composition at these energies is discussed by Stecker in his Erice lectures [i2] $]$. Protons interact with the CMBR, produce electron pairs, photoproduce pions and lose about $30 \%$ of their energy per interaction. This means that, unless protons are produced in our "cosmic backyard", they cannot have energies exceeding some $10^{19} \mathrm{eV}$ or so (the so-called GZK cutoff).

At the time of this writing some $20+$ events have been observed of energies exceeding the GZK cutoff and they have generated a flurry of theoretical papers.

All works submitted to this session dealing with the problem of trans-GZK cosmic rays contain a conjecture about some new physics or astrophysics to explain the existence of those events.

${ }^{*}$ Abridged version of the e-print hep-ph/0107095

${ }^{\dagger}$ Speaker. 


\section{Z-bursts and Neutrino Clouds.}

The original idea of Z-bursts was conceived by Fargion et al. and by Weiler, [4]. The basic idea is beautifully simple and it needs no particle physics beyond the well established Standard Model. Neutrinos can penetrate the CMBR because they have a very small magnetic moment and in scattering on a CMBR photon, the CMS energy, $\sqrt{s}$ is about $100 \mathrm{MeV}$ or so. There is a neutrino halo around the galaxy. If neutrinos are massive, an interaction between a neutrino coming from afar and a halo (anti) neutrino can produce a $Z$ resonance. The $Z$, in turn, decays into protons about $35 \%$ of the time generating trans-GZK cosmic rays.

Waxman pointed out that the total energy carried by the high energy neutrino flux was dangerously close to the total luminosity of the Universe [- Clustering or a high lepton asymmetry [i, $[\bar{i}]$ is needed in order to make the Z-burst scenario work. Unfortunately, a large lepton asymmetry leaves its imprint on the CMBR and the latest observations appear to

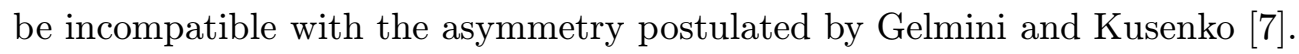

McKellar et al. [i in in a paper submitted to this meeting argue that clustering in the neutrino halo can be accomplished if one revives the idea of neutrino clouds [in]. The model is based on the assumption of a light scalar field weakly coupled to neutrinos. By performing a self consistent field calculation, the authors argue that the minimum of field energy is obtained if the neutrino beckground is not uniform, but clustered. This scenario has a chance to rescue the Z-burst model.

It is rather obvious that further research is needed in order to determine the viability of the neutrino cloud model. In particular, one should look for other measurable consequences of such a theory.

\section{Topological Defects, X-particles, etc.: the Saga of Top-Down Models.}

"Classic" (i.e. vintage $\simeq 1980$ 's) grand unified theories (GUTs) contain quite a few candidates of particles of masses around the GUT scale, ranging between (approximately) $10^{11} \mathrm{GeV}$ to $10^{16} \mathrm{GeV}$, the "X-particles". If sufficiently stable, they may be around us and decay, in part, into UHE protons, thus explaining the trans-GZK cosmic rays.

There is no known (weakly broken) symmetry to protect the X-particles from decaying rapidly. If this mechanism is to be a viable one, it is likely that the long decay lifetime is due to a higher dimensional operator as proposed in ref. [i] 100 . A recent detailed account of this approach can be found in ref. [i] $]_{1}^{1} \bar{i}$ with appropriate references to earlier work. Fodor and Katz [i] various theoretical considerations what the mass of the X-particle might be, they attempt to fit the available data by letting the mass of the X-particle float and be determined by the fitting procedure. In this way, they end up with a mass of $M_{X} \approx 10^{14.6} \mathrm{GeV}$ in contrast to the value, $M_{X} \approx 10^{12} \mathrm{GeV}$ used in ref. [i] leptoquark decaying into a quark and lepton would further increase the fitted $M_{X}$. Fodor and Katz point out that their fitted mass value leads to no contradiction with the observed 
X-ray and soft gamma ray background. However, should they consider other X-particles (like the leptoquark just mentioned) they may run into trouble with the EGRET results.

Another worry is based on the result of ref. [i] $\left.\overline{3}_{i}\right]$. As mentioned before, those authors find a geodesic GZK distance about a factor of 4 or so shorter than previously believed. Hence, the density of X-particles has to be increased by a factor of about 45 . Does this not overclose the Universe? Fodor and Katz assume a uniform distribution of X-particles. In reality, however, heavy X-particles cluster around galaxies, due to the gravitational attraction of the latter. Hence, the danger of overclosing the Universe is, perhaps, avoided.

\section{A String Inspired Model}

Internal consistency of string theories requires that strings live in a multidimensional space (e.g. $\mathrm{d}=10$ for superstrings). The connection between a string scale and the Planck scale

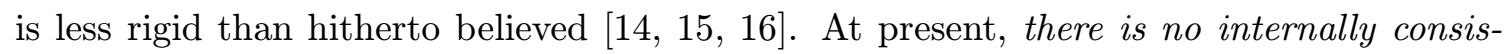
tent, phenomenologically viable string model known in which even the basic features of the dynamics - including a mechanism of compactification - would be satisfactorily understood.

Nevertheless, various string models have so many attractive properties that one is tempted to abstract their robust features and see whether some reasonable conjectures can be made once CMS energies of colliding particles reach the string scale. For the sake of argument, let us have a string scale of the order of a $100 \mathrm{TeV}$ in mind. This can be reached in UHE cosmic ray interactions: for instance, the "gold plated" Flye's Eye event has about $600 \mathrm{TeV}$ in the CMS.

Basically, three new phenomena are beginning to be observed around the string scale.

A large number of excitations begins to show up with, presumably, increasing widths. As a consequence, at least some cross sections exhibit a rapid rise towards a value which saturates unitarity. The excitations are, in essence, of two types.

Kaluza-Klein $(K-K)$ type excitations if the extra dimensions are compactified. Similar excitations may take place if we live on a brane and the extra dimensions are not compactified, see [i] $\overline{1} \overline{1}$ : there are brane fluctuations. The common feature of these excitations is that their level density grows like a power of the CMS energy; hence, at best, a cross section (for instance the $\nu N$ cross section) can grow only as a power of the energy. This is inadequate for explaining the trans-GZK cosmic rays.

The string excitations. The density of states in the excitation spectrum of strings grows asymptotically as $\exp (a \sqrt{s})$ for $s \gg M_{s}^{2}$. The low lying excitations exhibit a more rapid rise of the density of states, typically, $\propto \exp (b s)$, where $b$ is some model dependent constant.

There appears to be a unification of interactions. Once the energy gets into the string regime (after the first few excitations), all couplings are the same. We assume that the string and unification scales are the same, for lack of a really good model.

From the point of view of guessing a useful phenomenology, one conjectures that, in essence, there are three regimes of the future theory. 
The low energy regime characterized by the fact that coefficients of non renormalizable operators in an effective field theory, proportional to some positive power of $s / M_{s}^{2}$ are small.

The transition regime, in which $\mathrm{K}-\mathrm{K}$ excitations drive the coupling constants towards a common value.

The string regime proper, with all interactions unified and cross sections, in essence governed by their unitary limits.

It was pointed out some time ago [1] 18 is] that neutrinos could be ideal primaries of the trans-GZK cosmic rays since they have an essentially infinite mean free path in the CMBR. If one can arrange for a stronger than SM interaction with air nuclei, one could perhaps solve the puzzle of the trans-GZK events. The scenario sketched above provides the appropriate mechanism. In collisions with a CMBR photon, a neutrino of, say $E=10^{21} \mathrm{eV}$, has typically $\sqrt{s} \approx 200 \mathrm{MeV}$ or so: this is deep in the low energy regime even of the SM. By contrast, in interactions with a nucleon in an air nucleus, one has roughly $\sqrt{s} \approx 10^{3} \mathrm{TeV}$. Thus, we might be in the regime of unified interactions and string excitations [1 190

This scenario is predictive. One can easily understand in qualitative terms one of the most robust predictions $[2 \overline{2} \overline{0}]$. The dominant decay mode of the leptoquark excited in a $\nu$ nucleon interaction is a lepton and a quark. Therefore, the shower starts as if one had a lepton induced and a hadron induced shower running parallel to each other. The energy gets spread over many particles early in the development of the shower; hence, most of the evolution is governed by SM physics. The leptonic component develops by means of low multiplicity interactions ${ }^{1}$, the fluctuations are larger in a neutrino induced "anomalous" shower than in a proton (or nucleus) induced one.

The ALPS ${ }^{2}$ Monte Carlo algorithm [2] $\left.{ }_{1}^{2}\right]$ was run both for proton induced showers and for "anomalous" ones. As expected, "anomalous" showers exhibit larger fluctuations in the electron number, see ref. [200̄in. Thus, given sufficient statistics, one can distinguish between proton and $\nu$ induced showers.

\section{Discussion.}

HiRes and AGASA will continue observing. The Pierre Auger observatory and the planned orbiting detectors will be functioning in the near future. Hence, the event rate of trans-GZK showers is expected to be in the thousands per year. All scenarios submitted to this meeting (and others not submitted) have some advantages and disadvantages and various degrees of falsifiability Perhaps the scheme discussed last represents the most radical departure from established Standard Model physics. At the same time, it has the highest degree of falsifiability. Unfortunately, the calculations are very difficult: as shown by Cornet et al [i2̄2i], weakly coupled string theories in the tree approximation are unlikely to explain trans-GZK phenomena. Most probably, we'll have to learn how to handle strongly coupled string theories - or whatever will supercede them.

\footnotetext{
${ }^{1}$ Counting electrons and photons on the same footing, the average multiplicity in a lepton - nucleus interaction is very close to 2 .

${ }^{2}$ Adaptive Longitudinal Profile Simulation
} 
Let us end with an optimistic conclusion. We may have seen hints at physics beyond the Standard Model or at least, some interesting new astrophysics. The jury is still out on what the correct explanation is.

We thank D. Fargion, B. McKellar, S. Sarkar and T. Weiler for useful comments and discussions.

\section{References}

[1] K.G. Greisen, Phys. Rev. Letters 16, 748 (1966); G.T. Zatsepin and V.A. Kuzmin, JETP Letters (in Russian), 4, 144 (1966).

[2] F.W. Stecker, e-Print Archive astro-ph/0101072 (2001).

[3] D.J. Bird et al Phys.Rev. Letters 71, 3401 (1993). D.J. Bird et al. Ap. J. 424, 491 (1994).

[4] D. Fargion, B. Mele and A. Salis, Ap. J 517, 725 (1999); T.J. Weiler, Proc. PASCOS98, ed. by P. Nath (World Scientific, 1999) and Astropart. Phys. 11, 303 (1999).

[5] E. Waxman, e-print astro-ph/9804023.hys.

[6] G. Gelmini and A. Kusenko, Phys. Rev. Letters 82, 5202 (1999).

[7] W.H. Kinney and A. Riotto, Phys. Rev. Letters 83, 3366 (1999).

[8] B.H.J. McKellar, M. Garbutt, G.J. Stephenson and T. Goldman, e-print hep-ph/0106123

[9] G.J. Stephenson, T. Goldman and B.H.J. McKellar, Int. J. Mod. Phys. A13, 2765 (1998).

[10] J. Ellis, J.L. Lopez and D.V. Nanopoulos, Phys. Lett. B247, 257 (1990).

[11] S. Sarkar and R. Toldrà, e-Print Archive hep-ph/0108098.

[12] Z. Fodor and S.D. Katz, Phys. Rev. Letters 86, 3224 (2001) and hep-ph/0105348 (2001).

[13] T. Stanev, A. Mücke, R.J. Protheroe and J.P Rachen, Phys. Rev. D62: 093005 (2000).

[14] J. Lykken, Phys. Rev. D54, 3693 (1996).

[15] I. Antoniadis, S. Dimopoulos and G. Dvali, Nucl. Phys. B516, 55 (1997).

[16] K.R. Dienes, E. Dudas and T. Ghergetta, Phys. Let. B436, 55 (1998).

[17] L. Randall and R. Sundrum, Phys. Rev. Lett. 83, 4690 (1999).

[18] G. Domokos and S. Nussinov, Phys.Letters B187, 372 (1987).

[19] G. Domokos, and S. Kovesi-Domokos, Phys. Rev. Letters, 82, 1366 (1999).

[20] G. Domokos and S. Kovesi-Domokos, W.S. Burgett and J. Wrinkle, JHEP 0107:017,2001 e-Print Archive: hep-ph/0011156

[21] P.T. Mikulski, dissertation (2000). Available at http://owl.gsfc.nasa.gov/mikulski-thesis.pdf

[22] F. Cornet, J.I. Ilana and M. Masip, Phys. Rev Letters 86, 4235 (2001). 